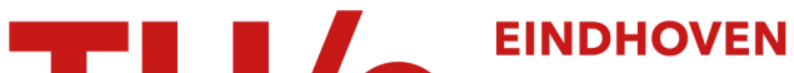 \\ UNIVERSITY OF \\ TECHNOLOGY
}

\section{High-capacity transmission over polymer optical fiber}

Citation for published version (APA):

Boom, van den, H. P. A., Bennekom, van, P. K., Tafur Monroy, I., \& Khoe, G. D. (2001). High-capacity transmission over polymer optical fiber. IEEE Journal of Selected Topics in Quantum Electronics, 7(3), 461-470. https://doi.org/10.1109/2944.962269

DOI:

10.1109/2944.962269

Document status and date:

Published: 01/01/2001

\section{Document Version:}

Publisher's PDF, also known as Version of Record (includes final page, issue and volume numbers)

\section{Please check the document version of this publication:}

- A submitted manuscript is the version of the article upon submission and before peer-review. There can be important differences between the submitted version and the official published version of record. People interested in the research are advised to contact the author for the final version of the publication, or visit the $\mathrm{DOI}$ to the publisher's website.

- The final author version and the galley proof are versions of the publication after peer review.

- The final published version features the final layout of the paper including the volume, issue and page numbers.

Link to publication

\section{General rights}

Copyright and moral rights for the publications made accessible in the public portal are retained by the authors and/or other copyright owners and it is a condition of accessing publications that users recognise and abide by the legal requirements associated with these rights.

- Users may download and print one copy of any publication from the public portal for the purpose of private study or research.

- You may not further distribute the material or use it for any profit-making activity or commercial gain

- You may freely distribute the URL identifying the publication in the public portal.

If the publication is distributed under the terms of Article 25fa of the Dutch Copyright Act, indicated by the "Taverne" license above, please follow below link for the End User Agreement:

www.tue.nl/taverne

Take down policy

If you believe that this document breaches copyright please contact us at:

openaccess@tue.nl

providing details and we will investigate your claim. 


\title{
High-Capacity Transmission Over Polymer Optical Fiber
}

\author{
H. P. A. van den Boom, W. Li, P. K. van Bennekom, I. Tafur Monroy, and Giok-Djan Khoe, Fellow, IEEE
}

\begin{abstract}
Polymer optical fiber (POF) is a promising transmission medium to provide broad-band telecommunication services within the customer's premises. POF offers several attractive features for data transmission such as broad bandwidth and low cost for in-house, access, and local-area-network (LAN) applications. This paper presents a review on optical transmission systems using POF and their enabling technologies. A summary is given of experimental data links with record capacity over record transmission distances. To conclude, we discuss trends for further development and research.
\end{abstract}

Index Terms-Optical communications, optical fiber, polymer optical fiber.

\section{INTRODUCTION}

$\mathbf{N}$ EW INTERACTIVE services require a broad-band communications network, which should extend into the customer's premises up to the terminals. At present, twisted pair and coaxial cables are used as the physical medium to deliver telecom services within the customer's premises. These two media suffer from serious shortcomings when they are considered to serve the increasing demand for broad-band services. For instance, twisted pair has a limited bandwidth and it is susceptible to electromagnetic interference (EMI). Coaxial cable offers a large bandwidth, but it poses practical problems due to its thickness and the effort required to make a reliable connection. Moreover, the coaxial cable is not immune to EMI.

Optical fiber is extensively used for long-distance data transmission and it represents an alternative for transmission at the customer premises as well. Optical fiber connections offer complete immunity to EMI. Optical silica-glass fibers, however, are not suitable for use within the customer premises because of the requirement of precise handling, and thus, the high costs involved. Polymer optical fibers are very attractive for use within the customer premises with their easy handling and low cost. This is mainly due to their relatively thick core. In fact, several polymer fiber-based systems are commercially available. However, these systems are based on the use of the multimode step index polymer optical fiber (SI-POF), whose bandwidth distance product is limited to a few $\mathrm{MHz} \cdot \mathrm{km}$.

The way toward broad-band POF systems is opened by the use of graded-index polymer optical fiber (GI-POF). The high bandwidth of the GI-POF (typically $2 \mathrm{GHz} \cdot \mathrm{km}[1]$ ) compared to SI-POF, is attributable to the graded-index profile in the core.

Manuscript received July 11, 2000; revised June 29, 2001.

The authors are with the COBRA Research Institute, Eindhoven University of Technology, Telecommunications Technology and Electromagnetics, $5600 \mathrm{MB}$ Eindhoven, The Netherlands (e-mail: i.tafur@tue.nl).

Publisher Item Identifier S 1077-260X(01)08936-5.

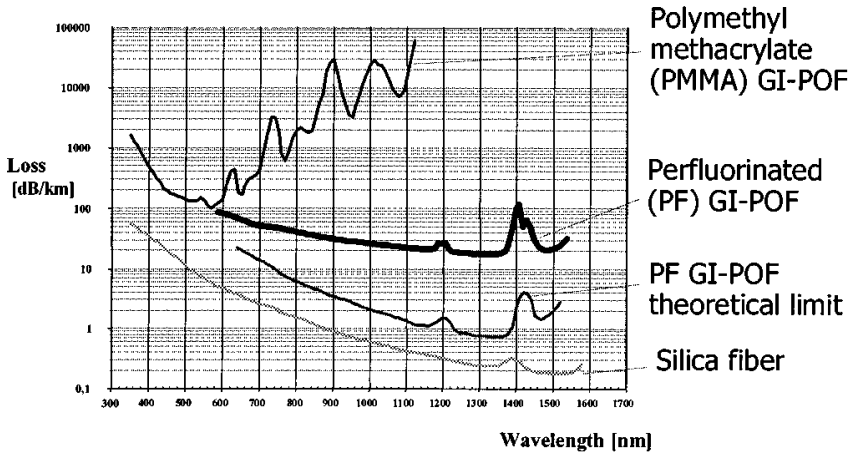

Fig. 1. Attenuation of graded index POF.

Additional characteristics of GI-POF are: 1) a large-core diameter (typically $500 \mu \mathrm{m}$ ), which allows easy handling, thus the use of low-cost devices and interconnection devices (similar to SI-POF) and 2) low attenuation over a wide range of wavelengths, thus enabling the use of wavelength multiplexing for enhanced capacity.

This paper focuses on the result of transmission experiments with GI-POF. Single-channel systems with PMMA GI-POF, perfluorinated-based GI-POF, as well as wavelength-division-multiplexing (WDM) systems will be reported. For a comparison, experimental transmission systems using thick silica fiber are reported and discussed. Moreover, enabling technologies are reviewed, and finally, new trends for development and research are discussed.

\section{POF AS TRANSMISSION MEDIUM}

This section presents a review of the transmission characteristics of POF.

\section{A. Attenuation}

Fig. 1 displays the attenuation of several types of POF for different wavelengths. Experimental and theoretical curves, as well as the result for the standard silica fiber, are plotted for comparison. Polymethyl methacrylate (PMMA) has been generally used as the core material of commercially available stepindex POF. Its attenuation limit is approximately $100 \mathrm{~dB} / \mathrm{km}$ in the visible region. Therefore, the high attenuation of $\mathrm{POF}$ compared to the silica-based fiber has limited the POF data link length, even when the bandwidth characteristics are improved by the GI-POF. Transmission distances can be further extended by using the perfluorinated (PF) amorphous polymer base GI-POF, which has a low-loss wavelength region from 500 to $1300 \mathrm{~nm}$ (see Fig. 1). 


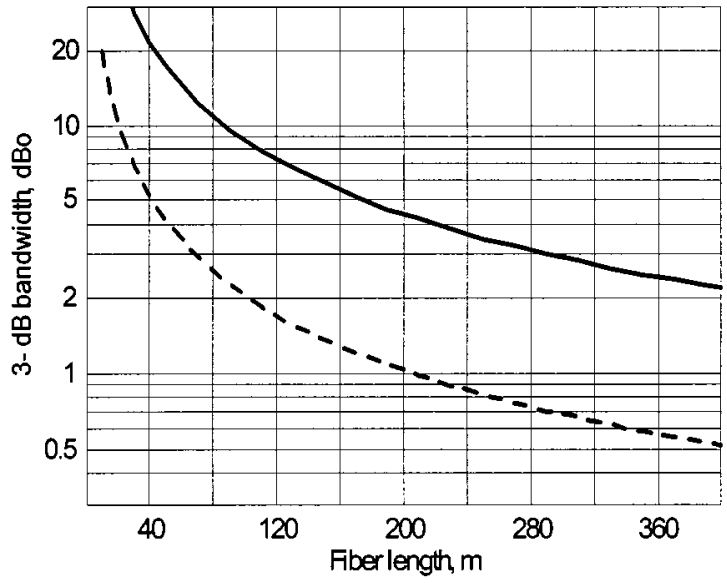

Fig. 2. Simulated bandwidth as a function of the fiber length: (-) Without the effect of DMA, $(---)$ With the effect of DMA.

We can observe in Fig. 1 that the experimental total attenuation of the PF polymer-based GI-POF decreases to $40 \mathrm{~dB} / \mathrm{km}$ even in the near infrared region. This means that $\mathrm{PF}$ fiber allows for the use of WDM channels for enhancing capacity.

\section{B. Bandwidth of $P O F$}

The advantage in bandwidth of the low material dispersion of PF polymer-based GI-POF has been theoretically and experimentally clarified in [2]. It has been shown that the low attenuation and low material dispersion of the PF polymer enables 1 - and $10-\mathrm{Gb} / \mathrm{s}$ transmission at 850 - and $1300-\mathrm{nm}$ wavelengths, respectively, as the PF polymer-based GI-POF has a very low material dispersion $(0.0055 \mathrm{~ns} / \mathrm{nm} \cdot \mathrm{km}$ at $850 \mathrm{~nm})$, as compared with the conventional PMMA-based POF, and compared with the multimode silica fiber $(0.0084 \mathrm{~ns} / \mathrm{nm} \cdot \mathrm{km}$ at $850 \mathrm{~nm})$.

Since the PF polymer-based GI-POF has a low attenuation compared with the conventional PMMA-based POF from the visible to the near infrared region, not only the 650 -nm wavelength region, which is the attenuation window of the PMMA base GI-POF, but also other wavelengths such as 850 or 1300 $\mathrm{nm}$, etc., can be used for transmission. It was clarified that the wavelength dependence of the optimum index profiles of the PF polymer-based GI-POF is very small, although the optimum index profile of the silica-based multimode fiber (MMF) at 650 $\mathrm{nm}$ differs greatly from that at $1300 \mathrm{~nm}$. This result indicates that the PF polymer-based GI-POF is very tolerant regarding the index profile for high-speed transmission as compared to multimode silica fiber. The impulse response function of the PF polymer-based GI-POF was accurately analyzed with the Wentzel-Kramer-Brillouin (WKB) numerical computation method using the measured refractive index profile. On considering all dispersion factors involving the profile dispersion, the predicted bandwidth characteristic of the PF polymer-based GI-POF agreed very well with the measurements. Fig. 2 presents the simulated bandwidth as a function of the fiber length, showing the effect of differential mode attenuation (DMA), which enhances the bandwidth significantly for the case of GI-POF. The mathematical formalism of the bandwidth model is presented in [3].

\section{TRANSMISSION EXPERIMENTS}

In this section, we present a review of transmission experiments using POF as the transmission medium. Their experimental setup and enabling technologies are described in detail. Several experiments have been performed to investigate the validity of theoretical models developed to predict the bandwidth of the fiber. The experiments have also shown the feasibility of POF links for high-capacity transmission.

A list of world record transmission experiments using POF can be found in Table I. Fig. 3 indicates the increase in transmission capacity. We denote the experiments carried out in our group by the TUE (Eindhoven University of Technology) tag.

\section{A. 2.5-Gb/s Transmission Over $200 \mathrm{~m}$ of PMMA GIPOF}

A $2.5-\mathrm{Gb} / \mathrm{s}$ system experiment over $100 \mathrm{~m}$, using a PMMA GI-POF, a visible light laser at $650-\mathrm{nm}$ wavelength and a silicon PIN photodiode has been reported earlier. In our experiment, the transmission distance is doubled to reach $200 \mathrm{~m}$. Key elements used in the experiment are a silicon avalanche photodiode (APD) receiver with a record sensitivity of $-29 \mathrm{dBm}$ at $2.5 \mathrm{~Gb} / \mathrm{s}$ and $10^{-9}$, a bit-error rate (BER) of PMMA GI-POF with a low attenuation of $0.164 \mathrm{~dB} / \mathrm{m}$ (Mitsubishi Rayon), and a laser (NEC) with a modulated spectral width of $0.4 \mathrm{~nm}$ at an average output power of $6.8 \mathrm{dBm}$. The experiment was carried out using a nonreturn-to-zero (NRZ) pseudorandom binary sequence (PRBS) with a pattern length of $2^{23}-1$. In Fig. 4, a block diagram of the experiment is shown.

One important feature in the APD receiver was the combination of the APD with two limiting amplifiers (HP) in a low-impedance front-end configuration. In principle, the APD can be set to a higher gain when combined with just one amplifier, but that will cause degradation caused by excess noise. Another significant improvement is the use of a data and clock recovery IC (Lucent Technologies) instead of using a direct clock connection and the decision circuit of the BER test equipment. To avoid electrical reflections, the APD limiting amplifiers and the clock recovery circuit were mounted as compact as possible.

Another key to the result is a new version of the GI-POF as compared to those reported earlier. The loss of the GI-POF has been decreased from 0.2 to $0.164 \mathrm{~dB} / \mathrm{m}$ by improving the homogeneity along the fiber by minimizing the fiber outer and core diameter variation, asymmetry of the index profile, and its change along the fiber. This has been achieved by a more precise control of preform preparation, drawing process, and cabling process.

Contributing to the achievement are measures taken to avoid reflections and to improve optical coupling efficiency between laser, fiber, and APD. Laser light is launched by means of a lens doublet that offers an numerical aperture (NA) of 0.55 at the laser side and 0.16 at the fiber side. At the receiver side, the lens doublet used has an NA of 0.25 at the fiber side and 0.55 at the APD side. The diameter of the active area of the APD was $230 \mu \mathrm{m}$. Coupling losses for both the laser side and the APD sides were less than $0.3 \mathrm{~dB}$. The lenses are antireflection (AR) coated to less than $1 \%$ reflection. The fiber end at the laser side was set at an angle of $4^{\circ}$ to avoid reflections into the laser. The coupling optics was manually adjusted to achieve a 
TABLE I

LIST OF EXPERIMENTAL TRANSMISSION RECORDS USING POF

\begin{tabular}{lccccc}
\hline \multicolumn{1}{c}{ Date } & Bitrate $[\mathrm{Gb} / \mathrm{s}]$ & $\begin{array}{c}\text { Distance } \\
{[\mathrm{m}]}\end{array}$ & Wavelength $[\mathrm{nm}]$ & $\begin{array}{c}\text { Fiber } \\
\text { material }\end{array}$ & Ref. \\
\hline 1994 & 2.5 & 100 & 650 & PMMA & {$[4]$} \\
1997 & 2.5 & 200 & 1300 & PF & {$[5]$} \\
$2-1998$ & 2.5 & 200 & 645 & PMMA & {$[6,7,8]$} \\
$8-1998$ & 5 & 200 & 1310 & PF & {$[9]$} \\
$10-1998$ & 2.5 & 300 & 645 & PF & {$[10]$} \\
$11-1998$ & 2.5 & 550 & 1310 & PF & {$[11]$} \\
$1-1999$ & 2.5 & 550 & 840 & PF & {$[11]$} \\
$2-1999$ & 11 & 100 & 1300 & PF & {$[12]$} \\
$9-1999$ & 7 & 80 & 950 & PF & {$[13]$} \\
$9-1999$ & $3 \times 2.5$ & 200 & $645,840,1300$ & PF & {$[14]$} \\
$3-2000$ & $2 \times 2.5$ & 328 & 840,1300 & PF & {$[15]$} \\
& & & & & \\
\hline
\end{tabular}

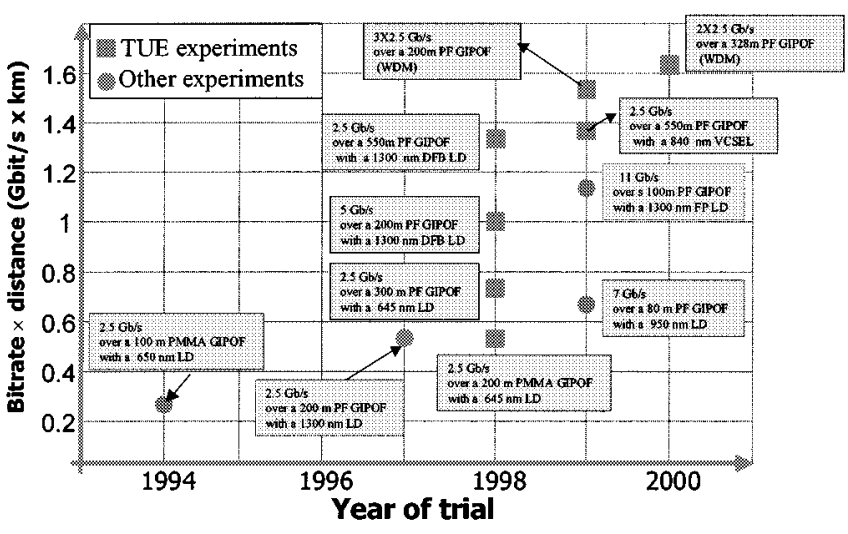

Fig. 3. Capacity increase of transmission systems using POF. Experimental trials.

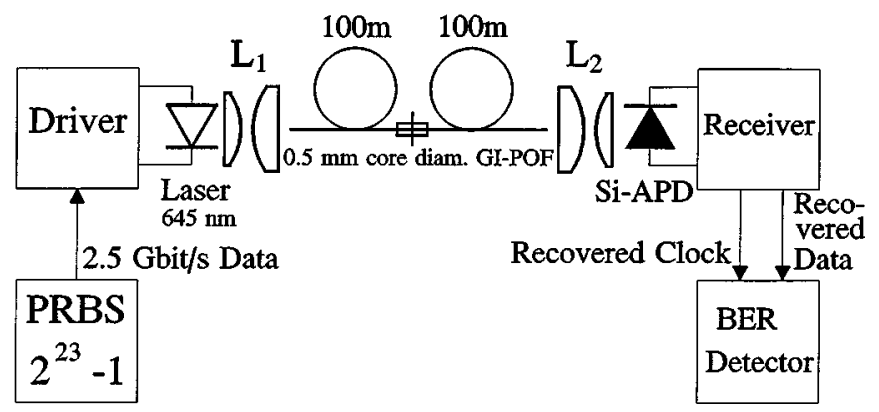

Fig. 4. 2.5-Gb/s transmission over $200 \mathrm{~m}$ of PMMA GI-POF.

maximum coupling efficiency. The eye diagram of back-to-back measurement and after 200-m of GI-POF are nearly identical, indicating a sufficient bandwidth (see Fig. 5).

The BER curve against received average power at the input of the APD receiver in the back-to-back and after $200 \mathrm{~m}$ GI-POF

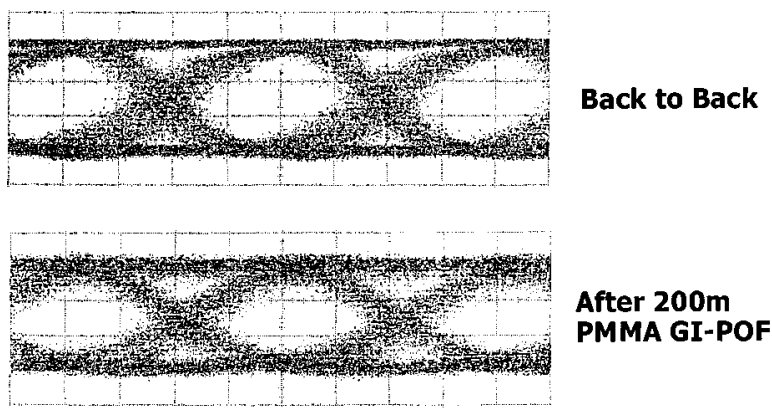

Fig. 5. Eye diagrams of back-to-back transmission and after 200-m GI-POF.

transmission has been measured (see Fig. 6). The back-to-back measurement has been carried out with a short piece of GI-POF of a few meters between transmitter and receiver. The received power has been changed by altering the distance between laser and GI-POF. The sensitivity of the receiver was $-29 \mathrm{dBm}$ at $2.5 \mathrm{~Gb} / \mathrm{s}$ for a BER of $10^{-9}$. The laser output power was $6.8 \mathrm{dBm}$, so the available power budget was $35.8 \mathrm{~dB}$.

The attenuation of the $2 \times 100 \mathrm{~m}$ GI-POF was $32.8 \mathrm{~dB}$. The power penalty due to modal dispersion of the fiber was $2 \mathrm{~dB}$ (see Fig. 6). The total coupling losses where $0.6 \mathrm{~dB}$, so a power budget of $35.4 \mathrm{~dB}$ was needed. Moreover, the optical output spectrum of the modulated laser at an average output power of $6.8 \mathrm{dBm}$ has been measured (see Fig. 7). The width of the spectrum, $3 \mathrm{~dB}$ below the maximum value is $0.4 \mathrm{~nm}$, which limits pulse broadening due to dispersion of the fiber.

It is of interest to analyze why our results outperform those reported so far. In addition to the receiver sensitivity, we believe that both the method of excitation, as well as the spectral characteristics of the exciting source play a significant role. Due to the tilting of the launching beam, back reflections from the fiber's input surface into the laser source are avoided. Semiconductor 


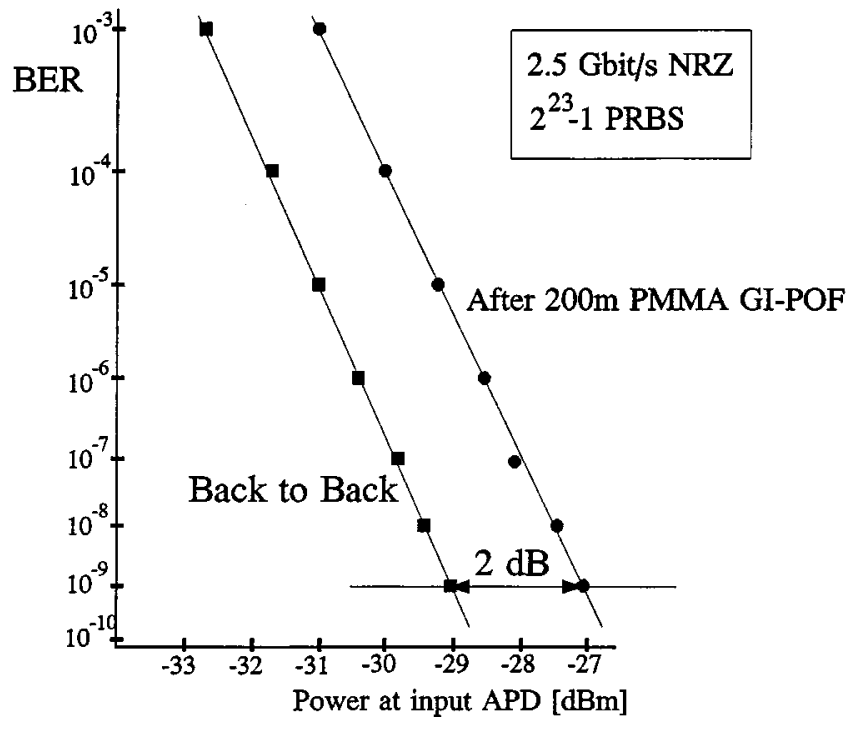

Fig. 6. BER measurement results.

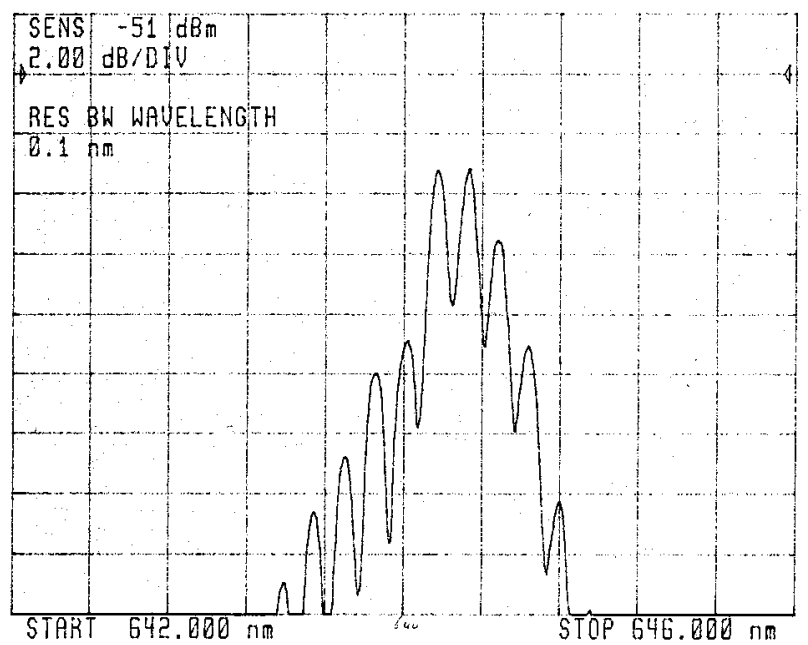

Fig. 7. Measured optical spectrum of the modulated laser.

lasers subject to back-reflected waves in optical communication systems may undergo different and complicated states of behaviors. Unless the reflected light is well monitored, optical feedback is often detrimental, because it enhances noise and introduces multiple nonlinearity in the emission characteristics, which degrade the signal-to-noise ratio at the receiver. Obviously, the fact that such an impairment was avoided could have contributed to the achievement of a good system BER. Another explanation to the present performance can be found in connection with the spectral characteristics of the exciting source. The measured spectral width was $0.4 \mathrm{~nm}$ as indicated earlier (see Fig. 7).

This small value certainly balances the effect of the material dispersion of the fiber. The influence of the wavelength has been checked by referencing to the existing literature. It can be seen that the bandwidth is significantly enhanced for $0.4 \mathrm{~nm}$ compared to the 2-nm case, as reported in the literature. The dispersion behavior of the fiber may be even better in the condition of our experiment. Indeed, the exciting beam was nearly parallel, meaning that the NA was not overfilled as in the case

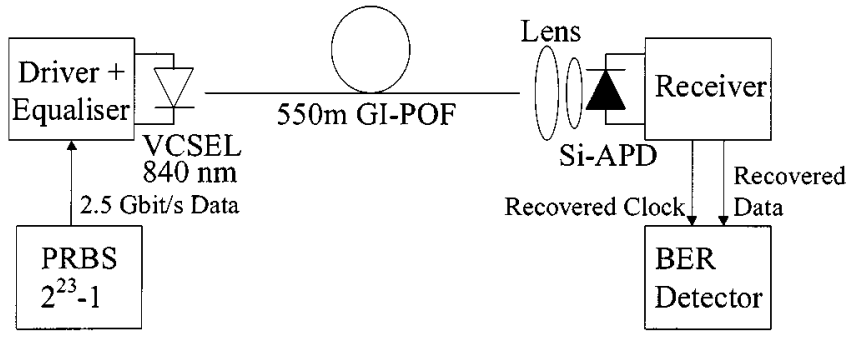

Fig. 8. Experimental setup for a PF GI-POF data link over $550 \mathrm{~m}$ at $840-\mathrm{nm}$ wavelength, using a VCSEL and a silicon APD.

of the curves presented in literature. So, the fiber should exhibit less modal dispersion, because the number of propagated modes is less than the one that can be excited under full launch condition, and this may lead to a further bandwidth improvement. The curves suggest that transmission experiments above 5 and $10 \mathrm{~Gb} / \mathrm{s}$ over $200 \mathrm{~m}$, respectively, may be possible if the fiber is graded around the optimum profile region $(g=2.33)$. Because the intrinsic bandwidth of the used laser was limited to $3 \mathrm{GHz}$, these experiments could not be carried out.

\section{B. 2.5- and 5-Gb/sTransmission Experiments With PF GIPOF}

At 840- and 1310-nm wavelengths, record distances of $550 \mathrm{~m}$ for both wavelengths have been achieved at a bit rate of $2.5 \mathrm{~Gb} / \mathrm{s}$. These results are obtained owing to the use of improved low-loss fiber, with very sensitive large active area APD receivers laser sources with a small spectral width, launching the fiber with only a few modes and improved optical interconnection between the components.

Perfluorinated polymer-based GI-POF has a low-loss wavelength region from 500 to $1300 \mathrm{~nm}$. Reported losses are $50 \mathrm{~dB} / \mathrm{km}$ from the visible to the near infrared region [13]. The $550 \mathrm{~m}$ of GI-POF we used, has an attenuation of $43.6 \mathrm{~dB} / \mathrm{km}$ at $840 \mathrm{~nm}$ and $31 \mathrm{~dB} / \mathrm{km}$ at $1310 \mathrm{~nm}$ (Asahi Glass). In our 2.5-Gb/s experiment at $1310 \mathrm{~nm}$, a transmission distance of $550 \mathrm{~m}$ has been reached by using a very sensitive large active area APD receiver and a low-loss interconnection between the GI-POF and the APD receiver. A 2.5-Gb/s experiment at $840 \mathrm{~nm}$ with GI-POF is, as far as we know, reported for the first time. In this experiment, a vertical-cavity surface-emitting laser (VCSEL) has been used in combination with a silicon APD receiver. Fig. 8 shows a block diagram of the 550-m distance experiment.

Key elements used in the 840-nm experiment are a VCSEL with a high bandwidth of $2 \mathrm{GHz}$, a silicon APD with a large active area of $230 \mu \mathrm{m}$ in diameter, and GI-POF with a low attenuation of $43.6 \mathrm{~dB} / \mathrm{km}$ at $840 \mathrm{~nm}$, as mentioned before. To compensate for the insufficient bandwidth of the VCSEL, an electrical equalizing circuit has been used. Key elements used in the 1310-nm experiment are a distributed-feedback (DFB) laser with a high bandwidth of $5 \mathrm{GHz}$, an InGaAs APD with an active area of $80 \mu \mathrm{m}$ in diameter, and GI-POF with a low attenuation of $31 \mathrm{~dB} / \mathrm{km}$ (Asahi Glass) at $1310 \mathrm{~nm}$, as mentioned before. Both experiments were carried out using a NRZ PRBS with a pattern length of $2^{23}-1$. Contributing to the achievement are measures taken to avoid reflections and to improve optical coupling efficiency between laser and fiber and fiber and 


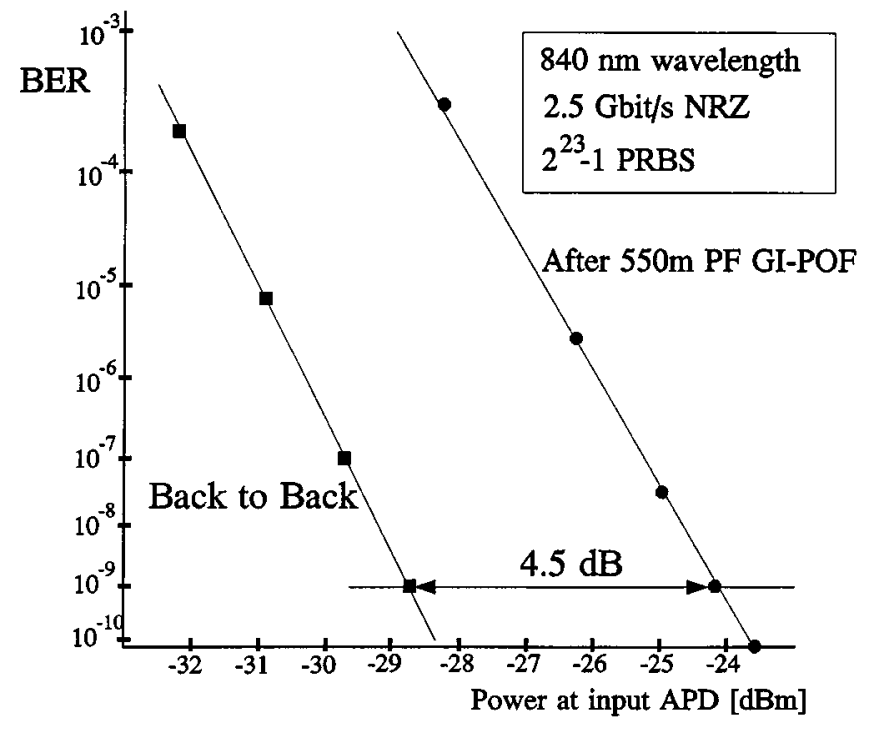

Fig. 9. BER $2.5 \mathrm{~Gb} / \mathrm{s}$ over $550-\mathrm{m}$ PF GI-POF at $840 \mathrm{~nm}$.

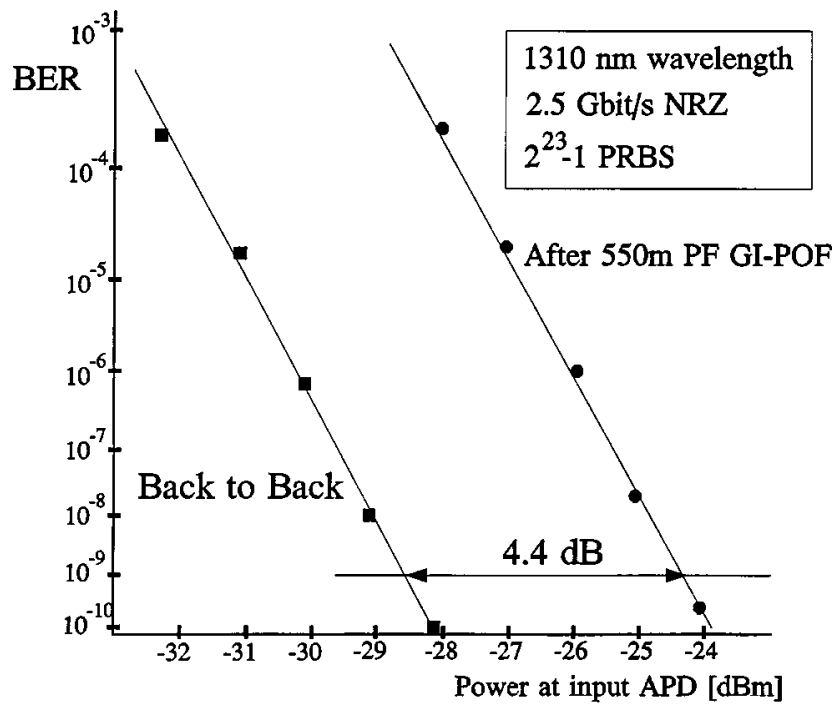

Fig. 10. BER $2.5 \mathrm{~Gb} / \mathrm{s}$ over $550-\mathrm{m}$ PF GI-POF at $1310 \mathrm{~nm}$.

APD. At the transmitting side in the 840-nm experiment, light is launched directly from VCSEL component into the GI-POF. Because the VCSEL component was already provided with a lens, which was not optimized for coupling with the GIPOF, the coupling loss was $1 \mathrm{~dB}$. In the 1310-nm experiment, the light from a single-mode fiber (SMF) pigtail of the DFB laser was launched into the large core of the GI-POF by means of a butt coupling with losses less than $0.1 \mathrm{~dB}$. At the receiver side in both experiments, a lens doublet has been used that has an NA of 0.25 at the fiber side and 0.55 at the APD side. GI-POF to APD coupling losses were less than $0.3 \mathrm{~dB}$. The lenses are AR-coated to less than $1 \%$ reflection. The coupling optics was manually adjusted to achieve a maximum coupling efficiency.

The BER as a function of received average power at the input of the APD has been measured back-to-back, and after 550-m GI-POF transmission (see Figs. 9 and 10). The back-to-back measurements has been carried out with a short piece of GI-POF of a few meters long between transmitter and receiver. In the

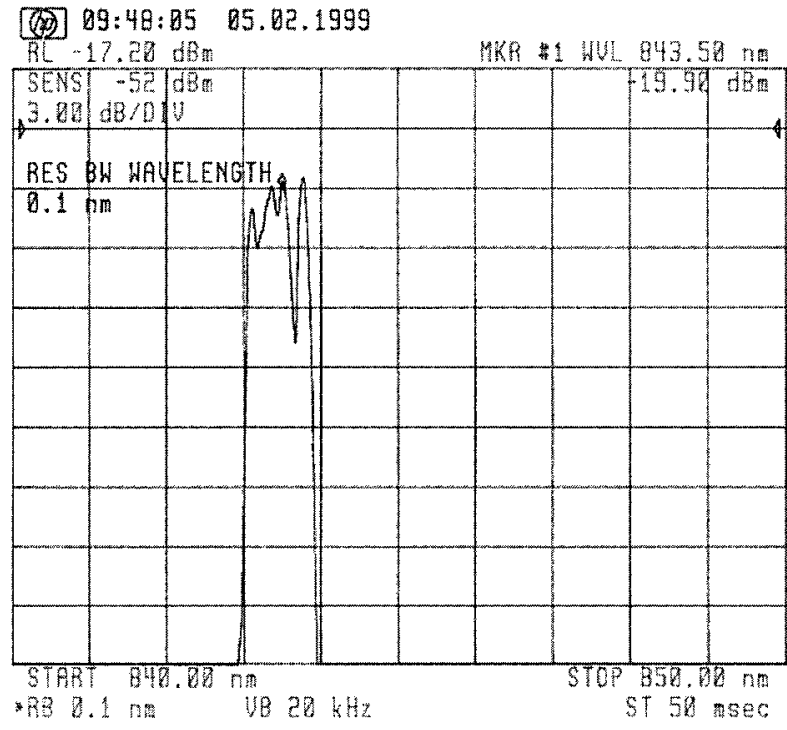

Fig. 11. Optical spectrum of modulated 840-nm VCSEL.

case of the 840-nm system, the received power has been changed by altering the distance between laser and GI-POF. In the case of the 1310-nm system, a variable attenuator has been used after the laser diode.

In the 840-nm experiment, the average output power of the VCSEL was $1.3 \mathrm{dBm}$, and the sensitivity of the receiver was $-28.6 \mathrm{dBm}$ at $2.5 \mathrm{~Gb} / \mathrm{s}$ for a BER of $10^{-9}$, so the available power budget was $29.9 \mathrm{~dB}$. The attenuation of the 550-m GI-POF was $24.0 \mathrm{~dB}$. The power penalty due to modal dispersion of the fiber and modal noise was $4.5 \mathrm{~dB}$ (see Fig. 9). The total coupling losses were $1.3 \mathrm{~dB}$, so a power budget of $29.8 \mathrm{~dB}$ was needed.

In the 1310-nm experiment, the average output power of the DFB laser was $0.4 \mathrm{dBm}$ and the sensitivity of the receiver was $-28.4 \mathrm{dBm}$ at $2.5 \mathrm{~Gb} / \mathrm{s}$ for a BER of $10^{-9}$, so the available power budget was $28.8 \mathrm{~dB}$. The attenuation of the $550-\mathrm{m}$ GI-POF was $16.3 \mathrm{~dB}$ at $1310 \mathrm{~nm}$. The power penalty, due to modal dispersion of the fiber and modal noise, was $4.4 \mathrm{~dB}$ (see Fig. 10). The total coupling losses were again $0.4 \mathrm{~dB}$, so a power budget of $21.1 \mathrm{~dB}$ was needed. Because of the difference between available and needed power of $7.7 \mathrm{~dB}$, a probable distance of $750 \mathrm{~m}$ can be reached. This experiment could not be carried out because this length of fiber was not available.

The modulated spectral width of the 840-nm laser was smaller than $1 \mathrm{~nm}$ (see Fig. 11) and smaller than $0.1 \mathrm{~nm}$ for the 1310-nm laser source. These small values certainly balance the effect of the material dispersion of the fiber. The dispersion is further avoided by the launching condition of our experiments. In case of the 1300-nm experiments, the SMF pigtail of the laser source was butt jointed to the GI-POF exciting only a few modes. In case of the 840-nm experiment, only a few modes are excited because the exciting beam was nearly parallel, meaning that the NA was not overfilled. So, the fiber should exhibit less modal dispersion because the number of propagated modes is less than the one that can be excited under full launch condition, and this may lead to a further bandwidth improvement.

A PF-GI-POF $2.5-\mathrm{Gb} / \mathrm{s}$ transmission system at $645-\mathrm{nm}$ visible light with a distance of $300 \mathrm{~m}$ has been carried out. For 


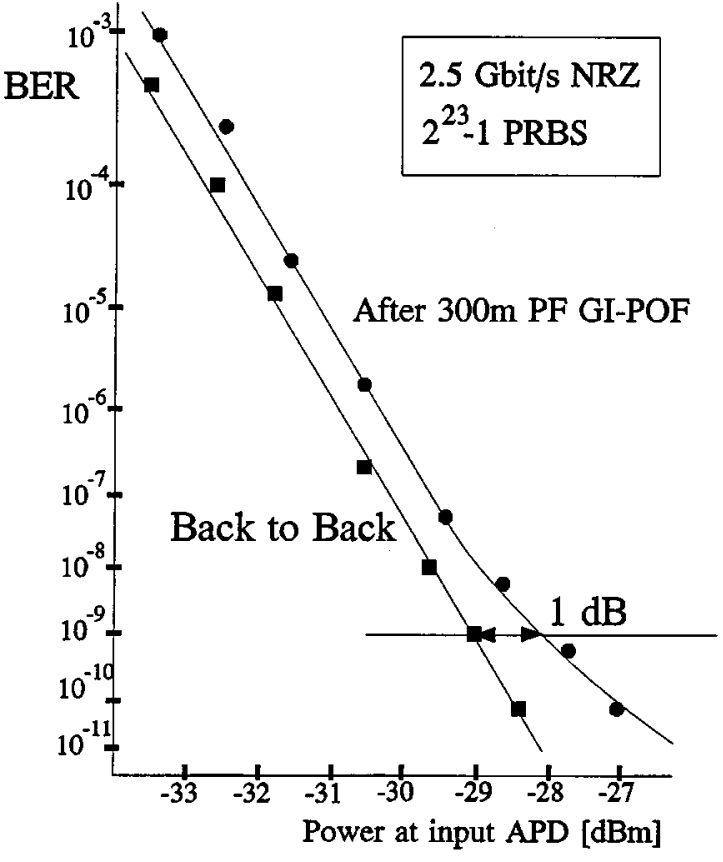

Fig. 12. BER of $2.5 \mathrm{~Gb} / \mathrm{s}$ over $300-\mathrm{m}$ PF GIPOF at $645 \mathrm{~nm}$.

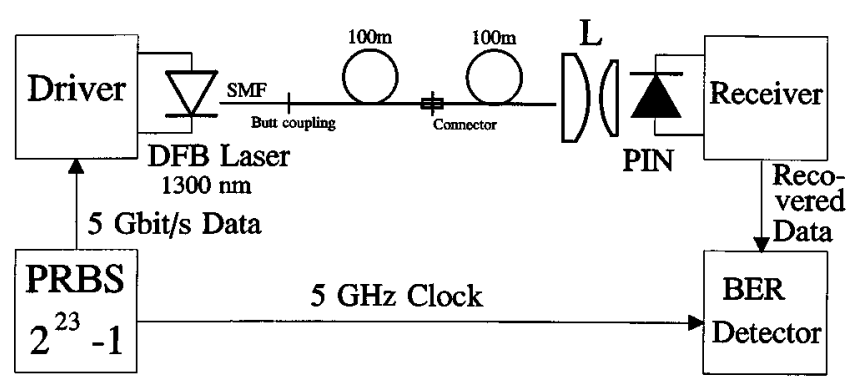

Fig. 13. $5 \mathrm{~Gb} / \mathrm{s}$ over 200-m PF GIPOF set up.

this experiment, the transmitter and receiver modules of the $2.5 \mathrm{~Gb} / \mathrm{s}$ over 200-m PMMA GIPOF have been used. Fig. 12 shows the measured BER against received average power at the input of the APD with and without 300-m GI-POF. Again, the back-to-back measurement has been carried out with a short piece of GI-POF transmitter and receiver. The sensitivity of the receiver was $-29 \mathrm{dBm}$ at $10^{-9} \mathrm{BER}$. The laser output power was $6.2 \mathrm{dBm}$, so the available power budget was $35.2 \mathrm{~dB}$. The attenuation of the $3 \times 100 \mathrm{~m}$ GI-POF was $32.6 \mathrm{~dB}$. The power penalty due to modal dispersion of the fiber and modal noise was $1 \mathrm{~dB}$ (see Fig. 12). The total coupling losses where $0.6 \mathrm{~dB}$, so a power budget of $34.2 \mathrm{~dB}$ was needed.

With the set up of Fig. 13, an error-free transmission experiment at a bit rate of $5 \mathrm{~Gb} / \mathrm{s}$ has been carried out over 200-m PF GIPOF at $1300 \mathrm{~nm}$.

Using a metal-semiconductor-metal (MSM) detector, developed by the Electronic Devices Group, Eindhoven University of Technology, a $2.5 \mathrm{~Gb} / \mathrm{s}$ over 100-m GIPOF has been carried out at $840 \mathrm{~nm}$. The MSM detector has a large active area of $100 \times 100 \mu \mathrm{m}$, and is therefore, easy to couple with a large-core fiber. The MSM detector was coupled with an amplifier with an input impedance of $50 \mathrm{ohms}$. The receiver sensitivity was -6

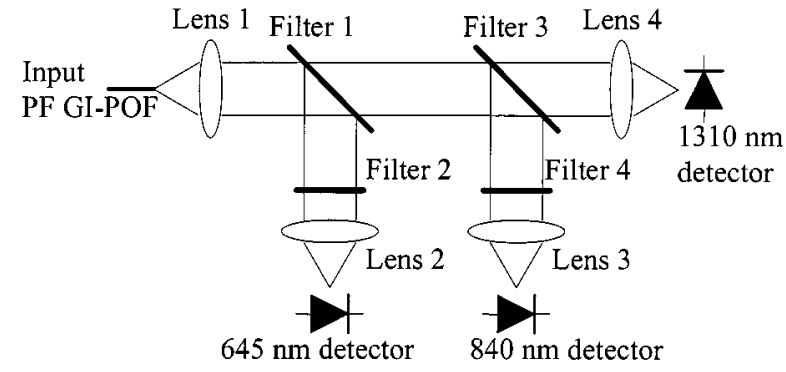

Fig. 14. Principle of operation of the 645-, 840-, 1310-nm demultiplexer.

$\mathrm{dBm}$ for a BER of $10^{-9}$. This sensitivity can be improved by using a transimpedance amplifier.

\section{WDM Experiments}

Perfluorinated polymer-based graded index polymer optical fiber (GI-POF) has a low-loss wavelength region from 500 to $1300 \mathrm{~nm}$, so many WDM transmission can be applied over a broad wavelength range, which can be separated easily with low-cost devices. As a start of this development, a demultiplexer for splitting up the wavelengths 645,840 , and $1310 \mathrm{~nm}$ has been realized with planar interference filters [17]. In Fig. 14, the principle of operation of the demultiplexer is shown.

First, the light from the input GI-POF is transformed into a parallel beam by means of lens 1 . Interference filter 1 deflects the light in the $645-\mathrm{nm}$ wavelength region. The other wavelengths are passed through. Second, to decrease crosstalk, an extra filter 2 has been used, which is only transparent for the 645-nm wavelength region. Lens 2 focuses the light at the photodiode of the 645-nm receiver. The light in the 840- and 1310-nm wavelength regions, which passed through filter 1, is split up by filter 3 Light in the 840-nm wavelength region is deflected by filter 3, filtered by filter 4 , and focused on the detector of the 840 -nm receiver by lens 3 . The remaining 1310 -nm light is focused on the 1310-nm detector by lens 4 . The measured insertion losses for all three wavelengths from GI-POF input to photo detectors are smaller than $1.6 \mathrm{~dB}$. Measured crosstalk levels are smaller than $-30 \mathrm{~dB}$. In Fig. 15, a photograph of the demultiplexer including the three receiver modules is shown. On the left, the GI-POF can be seen.

The demultiplexer has been used for a three channels operating at $2.5 \mathrm{~Gb} / \mathrm{s}$ over 200-m GI-POF WDM experiment with a record bit rate times distance product. A block diagram of the setup is shown in Fig. 16. For this experiment, the transmitters and receivers described in Section III A and Section III B have been used. Moreover, a two-channel at $2.5 \mathrm{~Gb} / \mathrm{s} \mathrm{WDM} \mathrm{experi-}$ ment has been carried out, using the wavelengths 840 and 1310 $\mathrm{nm}$, and a GI-POF fiber with a length of $328 \mathrm{~m}$ of one piece. Because this fiber sample has an attenuation of more than 100 $\mathrm{dB} / \mathrm{m}$ at $640 \mathrm{~nm}$, this wavelength could not be used. A block diagram of this setup is shown in Fig. 17.

\section{Experiments With Large-Core Silica Graded Index Fibers}

Present silica graded index multimode (GIMM) fibers are mainly produced in two types: 50/125 and 62.5/125 $\mu \mathrm{m}$. These fibers show a high modal bandwidth, certainly when produced 


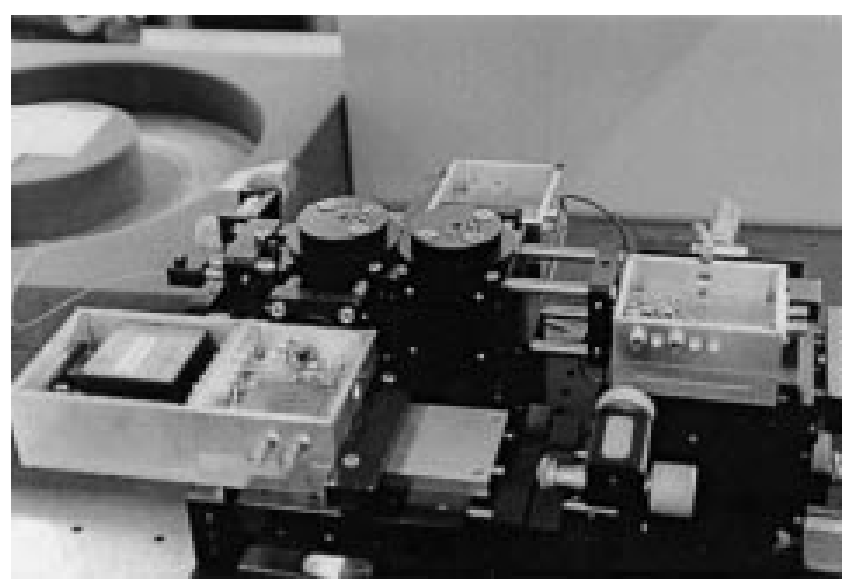

Fig. 15. Photograph of the realized demultiplexer including the three receiver modules.

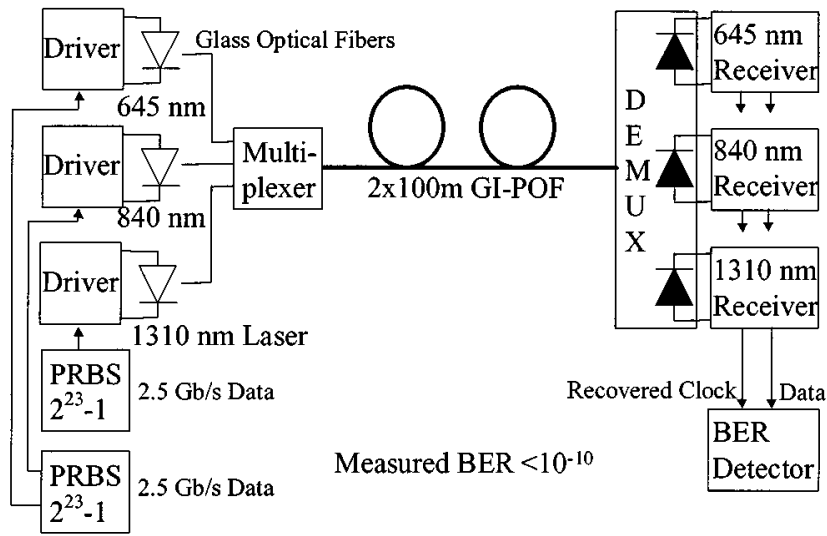

Fig. 16. Block diagram of the $3 \times 2.5-\mathrm{Gb} / \mathrm{s}$ WDM experiment over $200-\mathrm{m}$ GI-POF.

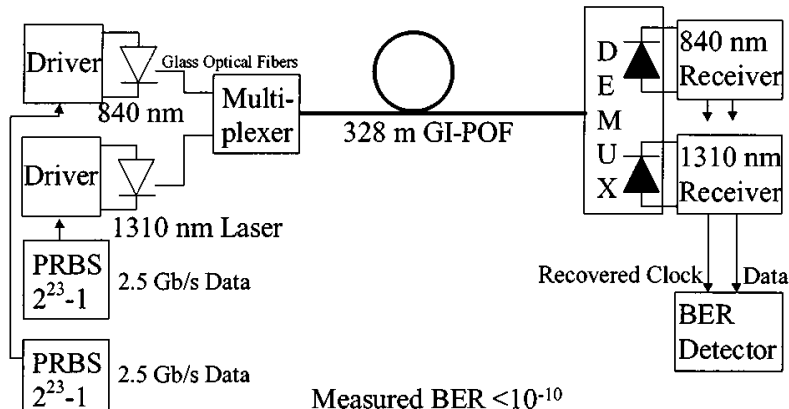

Fig. 17. Block diagram of the $2 \times 2.5-\mathrm{Gb} / \mathrm{s}$ WDM experiment over $328-\mathrm{m}$ GI-POF.

by for that purpose excellently equipped by the plasma chemical vapor deposition (PCVD) process. The quality of the profile shape is very important in relation to bandwidth. Although the profile shapes of GI-POF are not perfect, some reports refer to high bandwidth values. From this point of view, it is interesting to know the bandwidth behavior of large-core silica GIMM fibers, because that technology is available and present attenuation values are by far better than those of present PMMA GI-POFs. A series of investigations have been made using core sizes of 93,148 , and $185 \mu \mathrm{m}$. These fibers have been made from a standard 50- $\mu \mathrm{m}$ GIMM PCVD preform, drawn to different

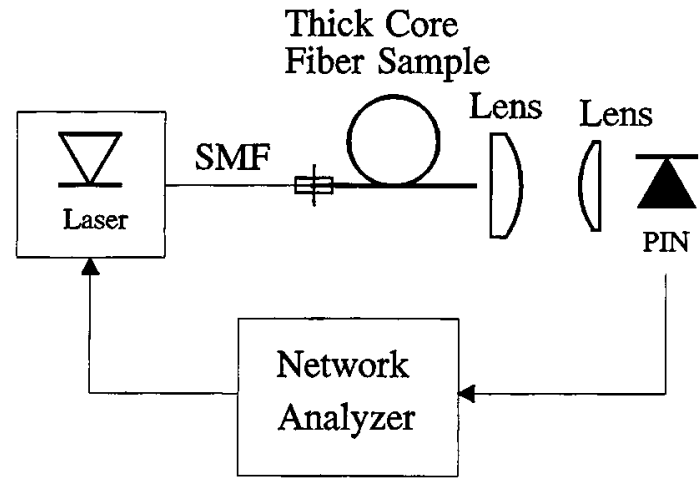

Fig. 18. Bandwidth measurement setup with network analyzer.

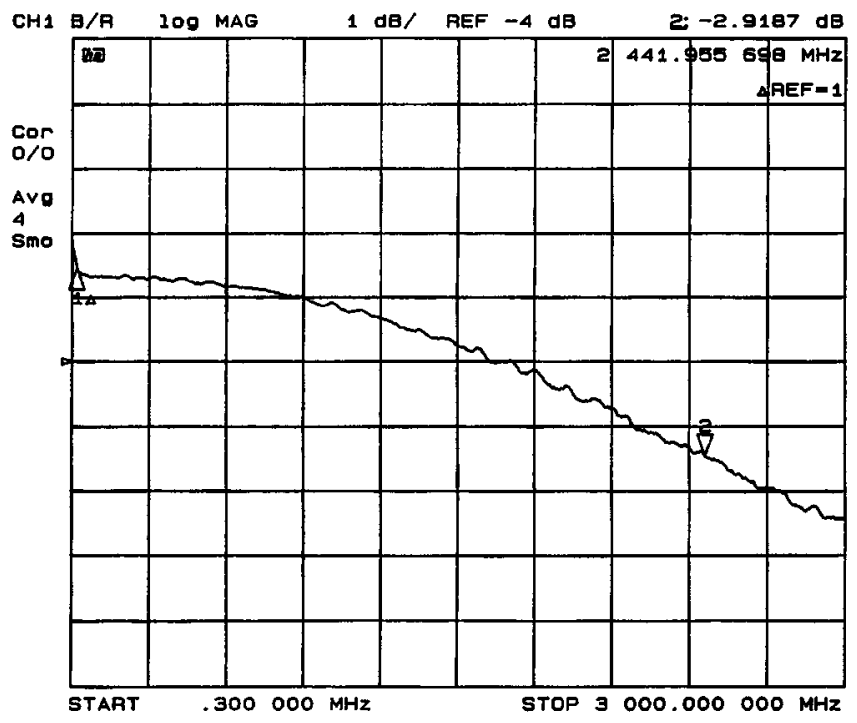

Fig. 19. Example of a result of a bandwidth measurement for a silica large-core graded-index MMF.

cladding diameters $(125,200$, and $250 \mu \mathrm{m}$, respectively). With these large-core fibers, it is impossible to excite all transmission modes at the transmitter side with a laser to obtain an over-filledlaunch (OFL) condition. This condition gives a worstcase bandwidth. The bandwidth can be increased by excitation of a small number of low-order modes, using a launch from an SMF. This technique has been used while performing the bandwidth measurements, transmission experiments, and taken into account in the theoretical analysis.

Using SMF launching, the bandwidth of the three fibers with a core/cladding diameter of 93/125, 148/200, and 185/250, each with a length of $2 \mathrm{~km}$, has been measured with a 3-GHz optical network analyzer at $1300-\mathrm{nm}$ wavelength (see Fig. 18). At the receiver side, a lens coupling has been used to detect all the power out of the fiber. The 3-dB bandwidths are 3.0, 2.4, and $2.3 \mathrm{GHz}$, respectively, and are comparable with the bandwidth of graded index fiber with standard diameters. An example of the result of a bandwidth measurement can be seen in Fig. 19.

Moreover, transmission experiments have been carried out at 1300-nm wavelength [18]. Again, a lens coupling has been used at the receiving end to detect all the power out of the fiber to avoid modal noise because of masking effects. The attenuation of the 2-km-long large-core GIMM is less than $1 \mathrm{~dB}$ at $1300 \mathrm{~nm}$, 
so the fiber introduces hardly any power loss. According to the bandwidth measurements and calculations, the bandwidth is sufficient, so for all three fibers at 2.5-Gb/s transmission, a BER smaller than $10^{-12}$ has been measured. Even with two combined 2-km fibers, so in total 4-km transmission distance, the BER was smaller than $10^{-12}$. To our knowledge, these $2.5-\mathrm{Gb} / \mathrm{s}$ transmission results over large-core graded index fiber are reported for the first time. A simulation model of the bandwidth of these fibers including limited fiber launching, mode coupling, chromatic dispersion, and differential mode attenuation has been developed. The measurement results are accordingly agreement with the simulation results.

\section{POTENTIALS}

Plastic optical fibers offer the potential to serve as the transmission medium for broad-band telecommunications service, for instance, in in-home networks and for local area network (LAN) networking environment. There are several alternative technologies such as copper-based silica SMF, and multimode glass fiber technologies. However, these technologies suffer from several drawbacks. Let us examine the advantages and disadvantages of POF with respect to those technologies. Compared to copper-based technologies, like coax cables and twisted pair, POF guarantees electromagnetic compatibility (EMC) and absence of crosstalk. Polymer optical fiber has smaller volume, it is less bulky, more flexile, and it has smaller weight. For data transmission, POF offers higher bandwidth at longer transmission distances. Compared to multimode glass optical fiber, POF is easier to handle. POF termination can be realized faster and cheaper than in the case of multimode glass fiber. The typical large core of polymer fiber allows for large tolerance on misalignments that results in the possibility of making cheaper connectors. For comparison, let us examine the power loss due to lateral (axial) misalignment of connecting two graded index (parabolic case) MMF with different core diameters. Calculations, assuming uniform modal power distributions for a misalignment of $25 \mu \mathrm{m}$, yields a loss of $1.76 \mathrm{~dB}$ for a $62.5-\mu \mathrm{m}$ core diameter MMF. For the case of PF GI_POF with a core diameter of $200-\mu \mathrm{m}$, the $25-\mu \mathrm{m}$ displacement results only in $0.48-\mathrm{dB}$ loss. Another advantage of large-core PF GI-POF has been observed with respect to the bandwidth degradation due to modal noise at misalignments in fiber-to-fiber connections. Namely, PF GI-POF shows very short time delay at the wide area of the core. This is contrast to the case of multimode silica fiber with 50 - to $62.5-\mu \mathrm{m}$ core, where small displacements cause severe bandwidth degradation [24].

With respect to data transmission, POF has the potential of high bandwidth and less problems of modal dispersion. Although the attenuation and bandwidth characteristics of POF are inferior compared to standard SMF, POF offers the already mentioned advantages of easy of coupling, termination, and flexibility. In the case of standard SMFs, specialized precision mechanism has to be used for coupling and handling. We can see that POF data characteristics, at the current state-of-the art, do not pair those of standard SMF, however they are superior to those of copper-based technologies. Furthermore, the installation and termination of POF are easier and promise low costs compared to SMF and multimode glass fiber. It is worth noting that connector devices for POF are not only easier to assemble compared to those for single-mode glass fiber, but also easier than those for HF coax cabling. Termination of coax cabling requires more skilled handling, as improper termination will cause large loss and considerable increased ingress noise. This will result in a considerable degradation of the system performance. On the other hand, POF connectors can be assembled easily for instance using a low-cost plastic ferrule [24]. Coupling loss using the above method has been measured to be in the order of $0.8 \mathrm{~dB}$ at $850-\mathrm{nm}$ wavelength [24]. Large-core glass fiber shows lower attenuation than POF, however their core size is restricted to $200-\mu \mathrm{m}$ due to the inherent inflexibility of glass. In this situation, POF offers again advantages concerning easy handling and termination, and tolerance to misalignments.

Ethernet is a widely deployed networking technology. Gigabit ethernet standard operating at $1.25 \mathrm{~Gb} / \mathrm{s}$ supports a range of transmission lengths: $100 \mathrm{~m}$ over copper wire, $550 \mathrm{~m}$ over multimode glass fiber with a 50 - or $62.5-\mu \mathrm{m}$ core, and $5 \mathrm{~km}$ over SMF. Recent experiments performed in our group have successfully demonstrated $1.25-\mathrm{Gb} / \mathrm{s}$ ethernet transmission over PF GI-POF reaching a distance of $990 \mathrm{~m}$ with good BER performance. These experiments show that PF GI-POF can be used for gigabit ethernet applications, in short, to medium link distances. The transmission results presented in this paper are record experiments. They show the feasibility of POF for high-capacity transmission. The reported experiments use a receiver with APD photodetector, which is not a cost competitive option in relation to conventional PIN photodiode receivers. A receiver with a conventional less costly PIN photodiode may also be used at expenses of lower receiver sensitivity. Assuming a degradation of $11 \mathrm{~dB}$ in receiver sensitivity (16.9- $\mathrm{dBm}$ receiver sensitivity at $2.5 \mathrm{~Gb} / \mathrm{s}$ using a PIN detector has been reported in [4]) with respect to the APD receiver, we estimate the following reachable transmission distances at $2.5 \mathrm{~Gb} / \mathrm{s}$. At the 840-nm operating system over PF GI-POF with attenuation of $43.6 \mathrm{~dB} / \mathrm{km}$, a distance of $280 \mathrm{~m}$ could be feasible. For a system operating at $1310 \mathrm{~nm}$ with a fiber attenuation of $31 \mathrm{~dB} / \mathrm{km}$, a transmission distance of $430 \mathrm{~m}$ could be reached. However, for POF technology to be competitive in the customer's premises, it will have to operate with low-cost components. These include low-cost light sources, low-cost receiver modules, and low-cost WDM devices. We would like to remark that the transmission end shows less technical problem than the reception end. This also applies for the case of WDM transmission systems. The WDM multiplexing can be performed simply by butt joining the pigtailed fibers from the laser sources, for instance, as it has been reported in our experiments. It remains, therefore, of high importance for further development of POF-based transmission systems, the development of low-cost reliable transceiver modules, connector, and WDM (de)multiplexing devices. Light sources based on VCSELs are promising solutions. Perhaps the use of plastic/polymer lens system for fiber to the photodetector coupling could be introduced for the realization of compact and reliable receivers. These, among others, are challenging issues open for further research and development. 


\section{CONCLUSION}

The transmission distances of PF GI-POF-based systems are increasing very fast. At bit rates of $2.5 \mathrm{~Gb} / \mathrm{s}$, system spans of 300 $\mathrm{m}$ at $645-\mathrm{nm}$ wavelength, and $550 \mathrm{~m}$ at $840-$ and $1310-\mathrm{nm}$ wavelengths have been reached. Using WDM transmission, system capacities have been further enhanced. For instance, we have reported a three-channel $\times 2.5-\mathrm{Gb} / \mathrm{s}$ GI-POF WDM transmission over 200 -m experiment and a two-channel $\times 2.5 \mathrm{~Gb} / \mathrm{s}$ over 328-m experiment with record bit-rate distance products. These experiments show the feasibility of high-capacity transmission over POF. It also has been shown that $2.5-\mathrm{Gb} / \mathrm{s}$ transmission over $4 \mathrm{~km}$ of large-core (148 and $185 \mu \mathrm{m})$ graded index silica fiber can easily be realized. Maximum transmission distances of the large-core graded index silica fibers are much larger compared with the graded index polymer fibers. There is a large difference in attenuation between silica and polymer fibers. The diameter of silica fibers is limited because of the inherent inflexibility of glass materials. Because of the difference in mechanical properties of silica and polymer the handling techniques are different.

The experimental results resorted in this paper clearly show the applicability of graded index polymer optical fiber for customer premises and local area networks. We believe that the record results reported here are important milestones that may encourage the development of polymer fiber systems and networks.

\section{ACKNOWLEDGMENT}

The authors would like to acknowledge Y. Koike, Keio University, Yokohama, Japan, for providing the GI-POF samples. Dr. N. Yoshihara and Dr. M. Naritomi, Asahi Glass, Yokohama, Japan, are acknowledged for providing us with PF GI-POF samples. Dr. S. Yamazaki, NEC Corporation, Kawasaki, Japan, is acknowledged for making available 645-nm laser diodes. The author would like to acknowledge Dr. K. Nakamura and Dr. Y. Kawaharada, Mitsubishi Rayon, Hiroshima, Japan, for providing PMMA GI-POF samples. G. Kuyt, Plasma Optical Fiber, Eindhoven, The Netherlands, is acknowledged for providing samples of large-core silica fiber. Dr. A. Valster, JDS-Uniphase Netherlands, Eindhoven, The Netherlands, is acknowledged for making available laser diodes.

\section{REFERENCES}

[1] Y. Koike, T. Ishigure, and E. Nihei, "High-bandwidth graded-index polymer optical fiber," J. Lightwave Technol., vol. 13, pp. 1475-1489, July 1995.

[2] G. D. Khoe, Y. Yoike, T. Ishigure, P. K. V. Bennekom, H. P. A. V. D. Boom, W. Li, and G. Yabre, "Status of GIPOF systems and related technologies," in 25th ECOC'99 Conf., Sept. 26-30, 1999, pp. II/274-II/277. Invited.

[3] G. Yabre, "Influence of core diameter on the 3-dB bandwidth of gradedindex optical fibers," J. Lightwave Technol., vol. 18, pp. 668-666, May 2000.

[4] T. Ishigure, E. Nihei, S. Yamazaki, K. Kobayashi, and Y. Koike, "2.5 Gbit/s $100 \mathrm{~m}$ data transmission using graded-index polymer optical fiber and high speed laser diode at $650 \mathrm{~nm}$ wavelength," Electron. Lett., vol. 31, pp. 467-469, Mar. 1995.
[5] H. Imai, "Applications of perfluorinated polymer fibers to optical transmission," in Proc. 6th Int. Conf. Plastic Opt. Fibers Applicat. (POF'97), Hawaii, 1997, pp. 29-30.

[6] W. Li, G. Khoe, H. V. D. Boom, G. Yabre, H. de Waardt, Y. Koike, S. Yamazaki, K. Nakamura, and Y. Kawaharada, "2.5 Gbit/s transmission over 200 m PMMA graded index polymer optical fiber using A $645 \mathrm{~nm}$ narrow spectrum laser and a silicon APD," Microwave Optical Technol. Lett., vol. 20, pp. 163-166, Feb. 5, 1999.

[7] W. Li, G. Khoe, H. V. D. Boom, H. de Waardt, Y. Koike, S. Yamazaki, K. Nakamura, and Y. Kawaharada, "A 2.5 Gbit/s 200 m PMMA GI-POF transmission experiment with $645 \mathrm{~nm}$ visible light," in Proc. IEICE 1998 Commun. Society Conf., Kofu, Sept. 29-Oct. 2, 1998, p. 400.

[8] - "2.5 Gbit/s transmission over $200 \mathrm{~m}$ PMMA graded index polymer optical fiber using a $645 \mathrm{~nm}$ narrow spectrum laser," IEEE Proc. LEOS'98 11th Ann.Meeting, vol. 2, Dec. 3-4. IEEE, Piscataway, USA, 1998, pp. 297-298.

[9] H. P. A. V. D. Boom, W. Li, G. D. Khoe, G. Yabre, H. de Waardt, Y. Koike, M. Naritomi, and N. Yoshihara et al., "Record $2.5 \mathrm{Gbit} / \mathrm{s}$ perfluorinated polymer optical fiber transmission at $1300 \mathrm{~nm}$ wavelength using a large area APD and limited launching," Proc. Symp. IEEE/LEOS Benelux Chapter, Nov. 26, 1998. Univ. Gent, Gent, Belgium, 1998, pp. 53-56.

[10] W. Li, G. D. Khoe, H. P. A. V. D. Boom, G. Yabre, H. de Waardt, Y. Koike, M. Naritomi, N. Yoshihara, and S. Yamazaki, "Record $2.5 \mathrm{Gbit} / \mathrm{s}$ transmission via polymer optical fiber at $645 \mathrm{~nm}$ visible light," presented at the 7th International POF Conf.' 98 , Oct. 5-8, 1998. Postdeadline Paper.

[11] W. Li, G. D. Khoe, H. P. A. V. D. Boom, G. Yabre, H. de Waardt, Y. Koike, M. Naritomi, and N. Yoshihara, "Record $2.5 \mathrm{Gbit} / \mathrm{s} 550 \mathrm{~m}$ GI POF transmission experiments at 840 and $1310 \mathrm{~nm}$ wavelength," in 7th Micropt. Conf./Int. POF Conf.'99, July 14-16, 1999, pp. 60-63.

[12] G. Giaretta, W. White, M. Wegmueller, R. V. Yelamarty, and T. Onishi, " $11 \mathrm{~Gb} / \mathrm{s}$ data transmission through $100 \mathrm{~m}$ of perfluorinated gradedindex polymer optical fiber," in Proc. OFC/IOOC'99, Feb. 1999, pp. PD14-1-PD14-3.

[13] T. Schnitzer, F. Mederer, H. Unold, R. Jäger, M. Kicherer, K. J. Ebeling, M. Naritomi, and R. Yoshida, "7 Gb/s data rate transmission using InGaAs VCSEL at $\lambda=950 \mathrm{~nm}$ and perfluorinated GI POF," in 8th Int. POF Conf.'98, Chiba, Japan, July 14-16, 1999, pp. 209-212.

[14] G. D. Khoe, "Exploring the use of GIPOF systems in the $640 \mathrm{~nm}$ to 1300 nm wavelength area," in Proc. 8th Int. POF Conf., Chiba, Japan, July 14-16, 1999, pp. 36-43. invited.

[15] G. D. Khoe, W. Li, G. Yabre, H. P. A. van den Boom, and P. K. van Bennekom, "Progress in GIPOF systems and enabling technologies," in Int. Optoelectron. Symp. 2000, vol. 55, Japan, June 7-9, 2000, pp. 9-10. Invited.

[16] K. Koganezawa and T. Onishi, "Progress in perfluorinated GI-POF, LUCINA," in Proc. Int. Conf. Plastic Opt. Fibers Appl. (POF'2000), MA, Sept. 2000, pp. 19-21.

[17] W. Li, G. D. Khoe, and H. P. A. V. D. Boom, "A perfluorinated based graded index polymer optical fiber demultiplexer for 645, 840 and 1310 nm," in Symp. IEEE/LEOS Benelux Chapter, ISBN 2-9600 226-0-2, Mons, Belgium, Nov. 15, 1999, pp. 73-76.

[18] G. D. Khoe, W. Li, P. K. V. Bennekom, G. Yabre, H. P. A. V. D. Boom, H. de Waardt, A. H. E. Breuls, G. Kuyt, and P. J. T. Pleunis, "Gigabit/s transmission via large core graded-index silica optical fibers in comparison with graded index polymer optical fibers," in 7th MOC Conf., July 14-16, 1999, pp. 264-267.

[19] G. D. Khoe, L. Wei, H. P. A. van den Boom, H. de Waardt, and G. Yabre, "Progress in graded index polymer optical fiber systems," presented at the Proc. 3rd Optoelectron. Commun. Conf., ISBN 4-930 813-84-0, Chiba, Japan, July 12-16, 1998. Business Center for Academic Societies Japan, Tokyo, Japan, pp. 180-181.

[20] Y. Watanabe, Y. Takano, R. Yishida, and G. Kuijpers, "Transmission test results of perfluorinated GIPOF using commercially available transceivers," in 8th Int. POF Conf.' 99 , July 14-16, 1999, pp. 56-59.

[21] F. Fredricx, Ch. Bouchat, P. Vetter, H. P. A. V. D. Boom, and W. Li, "Polymer optical fiber for datacommunication: a feasibility study," in Symp. IEEE/LEOS Benelux Chapter, ISBN 2-9 600 226-0-2, Mons, Belgium, Nov. 15, 1999, pp. 115-118.

[22] G. D. Khoe, W. Li, P. K. van Bennekom, G. Yabre, H. P. A. van den Boom, H. de Waardt, A. H. E. Breuls, G. Kuyt, and P. J. T. Pleunis, "Gigabit/s transmission via large core graded-index silica optical fibers in comparison with graded index polymer optical fibers," in Proc. 8th POF Int. Conf.' 99, Y. Koike and Y. Ogura, Eds., Chiba, Japan, July 14-16, 1999. The Group of Microoptics, Optical Soc. of Japan, Tokyo, Japan, pp. 192-195. 
[23] G. D. Khoe, H. P. A. van den Boom, W. Li, and H. de Waardt, "Status of plastic optical fibers and related technologies," in Proc. 2nd Workshop Fiber Optical Passive Components, ISBN-88-87 237-08-5, Pavia, Italy, June 8-9, 2000, pp. 154-163.

[24] K. Koganezawa and T. Onishi, "Progress in perfluorinated GI-POF, Lucina," in Conf. Proc. Int. POF Technical Conf., Cambridge, MA, 2000, pp. 19-21.

H. P. A. van den Boom, photograph and biography not available at the time of publication.

W. Li, photograph and biography not available at the time of publication.

P. K. van Bennekom, photograph and biography not available at the time of publication.

Idelfonso Tafur Monroy was born in el Castillo (Meta) Colombia, in 1968. $\mathrm{He}$ received the M.Sc. degree in multichannel telecommunications engineering from the St. Petersburg State University of Telecommunications, (formerly Bonch-Bruevitch Institute of Telecommunications), St. Petersburg, Russia and the Technology Licentiate degree in telecommunication theory from the Royal Institute of Technology, Stockholm, Sweden, at the department of Signals, Sensor, and System, in 1992 and 1996, respectively. The licentiate thesis treated the performance analysis of optically preamplified receivers. He received the Ph.D. degree in electrical engineering from the Eindhoven University of Technology, The Netherlands, in 1999.

Currently he is a Postdoctoral Researcher in the area optical networking and communication systems at the Eindhoven University of Technology.
Giok-Djan Khoe (S'71-M'71-SM'85-F'91) was born in Magelang, Indonesia, on July 22, 1946. He received the Electrical Engineering degree from the Eindhoven University of Technology, Eindhoven, The Netherlands, in 1971.

From 1971 to 1972, he was with the FOM Institute of Plasma Physics, Rijnhuizen, The Netherlands, where is was involved with laser diagnostics of plasmas. In 1973, he joined the Philips Research Laboratories. He was appointed a Part-Time Professor and then Full Professor at the Eindhoven University of Technology, in 1983 and 1994, respectively,. He is currently the Chairman of the Department of Telecommunication Technology and Electromagnetics. His work has been devoted to single-mode fiber (SMF) systems and components. He has authored and co-authored more than 100 papers, invited papers, and books. He holds more than 40 U.S. patents.

G. Khoe has served in the IEEE/Lasers and Electro-optics Society (LEOS) Board of Governors, as European representative, vice president, and elected member. He is also a member of the Executive Committee of the IEEE Benelux Section. His professional activities include many conferences, where he has served in technical committees, management committees, and advisory committees as a member or chairman. He has numerous involvements in journal activities as Associate Editor or as member of the advisory board. In Europe, he is closely involved in Community Research Programs and Dutch national research programs, as participant, evaluator, auditor, and program committee member. He is one of the founders of the Dutch COBRA University Research Institute. His is one of three recipients of the prestigious "Top Research School Photonics" Grant that is awarded to COBRA by The Netherlands Ministry of Education, Culture, and Science, in 1998. He is a recipient of the MOC/GRIN Award in 1997. 